\section{Cureus}

Received 01/29/2018

Review began 06/27/2018

Review ended 07/01/2018

Published 07/08/2018

\section{(c) Copyright 2018}

Durrani et al. This is an open access article distributed under the terms of the Creative Commons Attribution License CC-BY 3.0., which permits unrestricted use, distribution, and reproduction in any medium, provided the original author and source are credited.

\title{
Breast Cancer During Pregnancy
}

\author{
Sajid Durrani ${ }^{1}$, Shomaila Akbar ${ }^{2}$, Humariya Heena ${ }^{3}$ \\ 1. Medical Oncology, King Fahad Medical City, Riyadh, SAU 2. Radiation Oncology, King Fahad Medical \\ City, Riyadh, SAU 3. Epidemiology and Public Health, King Fahad Medical City, Riyadh, SAU
}

$\square$ Corresponding author: Humariya Heena, hmunshi@kfmc.med.sa

Disclosures can be found in Additional Information at the end of the article

\section{Abstract}

Breast cancer remains the most common cancer in women. A diagnosis of cancer during pregnancy is uncommon. In recent decades, obstetricians are seeing an increasing number of women who become pregnant or desire to become pregnant after breast cancer treatment because of a delay in childbearing for a variety of reasons, including cultural, educational, and professional. Consequently, breast cancer in young women often occurs before the completion of reproductive plans.

A discussion among the patient, the oncologist, and the obstetrician on the relative benefits of early delivery followed by treatment versus commencement of therapy while continuing the pregnancy is of utmost importance in order to reach a consensual decision. The best available evidence suggests that pregnancy after breast cancer increases the risk of recurrence. The birth outcome in women with a history of breast cancer is no different from that in the normal female population; however, increased risks of delivery complications have been reported in the literature.

As concurrent pregnancy and breast cancer are uncommon, there are no data from large randomized trials; hence, recommendations are mainly based on retrospective studies.

Categories: Obstetrics/Gynecology, Oncology

Keywords: breast cancer, pregnancy, breast cancer treatment, breast cancer diagnosis

\section{Introduction And Background}

The incidence of breast cancer in pregnancy is approximately 1 in 3,000 and can reach up to 3\% [1]. The prevalence of pregnancy-associated breast cancer may be increasing owing to delayed childbearing, and despite its low incidence, breast cancer is the second most common cancer in pregnant women. In this review, we aimed to provide insights into the available diagnostic and therapeutic modalities for breast cancer during pregnancy and to elaborate on the prognosis and best practices for these concurrent conditions.

A case series conducted at Memorial Sloan-Kettering Cancer Center (New York, NY, USA) in 1991 revealed that 44 of 56 patients with breast cancer did not have cancer diagnosed until after delivery, although more than half of the women already had symptoms prenatally. It was observed that the women frequently presented with tumors having adverse pathological prognostic features (median tumor size, $3.5 \mathrm{~cm}$ ), suggesting either that the breast masses were not clinically recognized or that follow-up was delayed [2]. Moreover, many masses discovered during pregnancy and lactation are attributed to physiologic changes and likely to be discounted by the patient. 
The available data on pregnant patients with breast cancer are primarily from either case reports or case-control studies because of the ethical restrictions in conducting randomized clinical trials in such patients. Pregnant women with breast cancer are more likely to have larger tumors, positive nodes, metastases, and vascular invasion. In a retrospective casecontrol study, Zemlickis et al. found that breast cancer during pregnancy was more likely to be diagnosed at a later stage when distant metastasis had already occurred [3].

Bonnier et al. conducted an extensive study on hormone receptor status in 75 pregnant patients with breast cancer and a control group of 182 non-pregnant patients with breast cancer. Of the pregnant women, $56 \%$ to $83 \%$ had positive nodes at diagnosis versus $38 \%$ to $54 \%$ of the nonpregnant women. In the pregnant group, $42 \%$ of cancers were estrogen receptor (ER)-negative and progesterone receptor (PR)-negative, compared with $21 \%$ in the age-matched controls [4].

Many studies support the theory that ER-positive status is decreased in pregnant patients with breast cancer owing to receptor downregulation in pregnancy. Costa et al. hypothesized that high levels of circulating estrogens might even prevent ER-positive cancers [5].

\section{Review}

\section{Risk of breast cancer}

The medical literature has already established that nulliparous women have a higher risk of developing breast cancer than multiparous women [6]. Similarly, nulliparous women having their first child after 30 years of age are at a slightly higher risk of developing breast cancer than women who are usually exposed to higher estrogen levels over a prolonged period. Early menarche (before the age of 12 years) and menopause after the age of 55 years are also significant risk factors. Cyclical hormone levels also predispose women to breast cancer over time. This may be because breast cells grow and divide in response to hormones, such as estrogen, and pregnancy leads to an interruption of the normal cyclical hormone levels [7].

\section{Diagnosis and presentation}

Breast cancer in pregnancy most often presents as a painless mass or thickening in the breast occasionally associated with discharge from the nipple. About $95 \%$ of women in one study and $82 \%$ of women in another study presented with a painless mass [8]. Normally, the mean breast weight doubles in pregnancy from $200 \mathrm{~g}$ to $400 \mathrm{~g}$, resulting in increased firmness and density of the breast, which make the interpretation of the clinical examination and mammogram results more difficult. The hypertrophy and engorgement of the breasts may make both physical examination and mammographic imaging more challenging in pregnant than in non-pregnant patients. The preliminary investigation may be performed in non-pregnant women; however, for pregnant women, particular attention should be paid to the risks of ionizing radiation exposure for the fetus.

\section{Physical Examination}

All pregnant women should undergo clinical breast evaluation as part of the first physical examination. If a woman presents with a breast lump during pregnancy, she should be referred to a team of breast specialists.

\section{Mammography}

Although mammography with abdominal shielding can be performed with minimal risk during pregnancy, mammograms are difficult to interpret and hence, not advisable for the investigation of breast cancer in pregnant women [4]. A mammogram during pregnancy may 
reveal calcification, asymmetric density, axillary lymphadenopathy, and skin and trabecular thickening, which are helpful in the diagnosis of pregnancy-associated breast cancer [5].

\section{Ultrasonography}

Ultrasonography is a sensitive (100\% sensitivity in one series) investigation method in pregnant and lactating women based on three small series [8]. The most common sonographic finding is an irregular mass lesion with posterior acoustic enhancement. A marked cystic component is also seen occasionally. Ultrasonography is useful in detecting axillary metastases and in assessing response to systemic treatment in pregnant women with breast cancer [9]. Therefore, ultrasonography is the preferred imaging modality because of the radiodensity of the breast in the pregnant or lactating state. Ultrasonography can also aid in the assessment of the response to neoadjuvant chemotherapy. Hence, it is a simple and sensitive modality for the initial evaluation of breast cancer in pregnant and lactating women.

\section{Chest Radiography}

With adequate shielding, chest radiography is considered safe during pregnancy, as the expected fetal dose is less than the harmful threshold [10]. However, its use is recommended only when exclusively indicated.

Magnetic Resonance Imaging (MRI)

MRI will most often be the procedure of choice because it does not use ionizing radiation and can identify bone metastasis [11]; however, MRI is associated with risks to the fetus due to heating and cavitation [12]. Although some radiologists remain opposed to the use of MRI in the first trimester, there is currently no consensus on this matter. A United Kingdom (UK) medical device agency recommends that MRI should be avoided during the first trimester, as there is a theoretical risk of fetal damage due to the high magnetic field [13].

As the contrast agent (gadolinium) can cross the placenta and its effects on the developing fetus are unknown, its use should be avoided. Gadolinium is classified by the Food and Drug Administration (FDA) as a Pregnancy Category $\mathrm{C}$ drug and is associated with fetal anomalies [14]. Positron emission-computed tomography, computed tomography, and pelvic radiography involve more radiation than MRI, and hence are not the preferred imaging modalities [15-17]. Bone scans, although rarely used, result in only $0.00194 \mathrm{~Gy}$ of radiation exposure to the fetus. As a consensus in clinical practice, all staging investigations that are likely to cause any risk to the fetus should be done only where a positive result would alter immediate management (Level IV).

\section{Pathology}

Because normal breast tissue shows atypical cytomorphologic features during pregnancy and lactation, evaluation of aspiration samples is difficult [18-21]. Core needle biopsy may be an appropriate initial procedure with a sensitivity of 90\%; however, it increases the risk of milk fistula formation, infection, and bleeding [20-22].

Biopsy of a suspicious mass has been accepted as the gold standard for the diagnosis of breast cancer [23]. Appropriate risk reduction strategies include the use of prophylactic antibiotics and meticulous attention to hemostasis.

\section{Principles of treatment}


Treatment during pregnancy requires a discussion among the pregnant woman with breast cancer, the oncologist, and the obstetrician, on the relative benefits of early delivery followed by treatment versus commencement of therapy while continuing the pregnancy. Another factor preventing the administration of optimal therapy for the mother with breast cancer is the concern for the well-being of the fetus. In the selection of treatment, the factors considered at the time of diagnosis are the current stage of disease, hormone receptor status, and trimester of pregnancy. There should be a discussion about pregnancy and future fertility, and the patient should be informed that treatment for breast cancer may contribute to or cause infertility. The American Society of Clinical Oncology guideline highly recommends that an oncologist, along with a fertility specialist, counsel the patient about fertility preservation. Embryo and oocyte cryopreservation are both standard fertility procedures in most advanced centers worldwide, although their use is limited in developing countries.

\section{Surgery}

(1) First trimester: Surgery with mastectomy and axillary staging is recommended in the first trimester of pregnancy. Breast-conserving surgery (BCS) is not preferred in the first trimester because this technique requires radiation therapy, and radiation therapy has to be delayed during the first trimester. Breast reconstruction is also not an option in the first trimester; it should be delayed to limit the anesthetic exposure of the patient and the fetus as much as possible, as most patients require general anesthesia. Hence, pregnant women undergoing surgery under general anesthesia are more likely to give birth to low birth weight infants owing to prematurity and intrauterine growth retardation. Surgery is delayed up to the 12th week of gestation to avoid the high risk of spontaneous abortion, and the fetus is monitored according to gestational age. A study was conducted on 720,000 pregnant women among whom 5,405 underwent surgery, and it was found that there was an increased risk of neonatal mortality but no increase in congenital malformations and stillbirths [24]. Thus, surgery can be safely performed in patients with pregnancy-associated breast cancer with no unexpected complications.

(2) Second and third trimesters: Most of the patients in the published literature underwent mastectomy with axillary clearance. BCS with axillary dissection may be possible, especially in women in whom cancer is diagnosed in the second or third trimester; however, in women with advanced disease at presentation, neoadjuvant chemotherapy and surgery should be performed later in pregnancy or postpartum. The effect of sentinel node biopsy (SNB) has not been systematically evaluated in pregnant patients with breast cancer. SNB with isosulfan blue dye is not recommended for pregnant women, as anaphylaxis has been observed with the use of blue dye [25]. SNB with radioisotopes has been estimated to be associated with low radiation exposure to the fetus. Some have offered SNB after counseling the patient about its efficacy and safety because nodal metastases are commonly found in pregnancy-associated breast cancers and nodal status affects the choice of adjuvant chemotherapy [25]. The risks of SNB in pregnant women are unknown. Although some studies have reported the safety of SNB, data supporting this assertion are insufficient. For patients in whom cancer was diagnosed in the late second and third trimesters, lumpectomy and axillary dissection followed by irradiation after delivery are preferred over axillary dissection [26-29]. If the woman is far from term, BCS can be followed by chemotherapy after the first trimester and irradiation can be added after delivery. Modified BCS for Stage I and II tumors do not seem to have an advantage over modified radical mastectomy in terms of survival.

The common effects of pregnancy and cancer include dyspnea, nausea, and vomiting. The same treatment protocol is followed for these symptoms irrespective of the association with cancer or pregnancy. Factors that make anesthesia use difficult in pregnant patients include the hypercoagulable state, delayed gastric emptying, decreased functional residual capacity of the lungs, and increased blood volume and cardiac output. Therefore, adequate preoxygenation, 
rapid sequence induction with cricoid pressure, and other additional measures should be taken to prevent complications.

\section{Radiotherapy}

As any exposure to radiation may put the fetus at risk, adjuvant radiotherapy is usually delayed until after delivery.

\section{Chemotherapy}

(1) Maternal effects of chemotherapy: Physiological changes in pregnancy may alter the pharmacokinetic and pharmacodynamics of chemotherapy. These changes include alterations in hepatic metabolism, renal plasma flow, and plasma protein binding, all of which may affect drug clearance [30]. Amniotic fluid may act as a pharmacological third space and may delay the elimination of agents, such as methotrexate, resulting in increased toxicity.

(2) Fetal effects of chemotherapy: All drugs have the potential to cross the placenta depending on their physical and chemical properties. Chemotherapy can have several long-term effects, including gonadal dysfunction, germ-cell mutagenesis, teratogenicity in subsequent generations, and impaired physical and neurologic development.

(3) First trimester: When chemotherapy is given during the first trimester, there is a significant risk of spontaneous abortion and up to a $17 \%$ estimated risk of fetal malformations [10]. Hence, chemotherapy is avoided during this stage of pregnancy.

(4) Second and third trimesters: As organogenesis is complete at these stages, chemotherapy is widely used in the second and third trimesters. The only prospective series on this issue showed that there were no detectable congenital abnormalities and no maternal or fetal deaths in 24 pregnant women were treated with FAC (5-fluorouracil, Adriamycin, and cyclophosphamide) [9]. In another study of 28 patients who were treated with three different regimens (doxorubicin and cyclophosphamide; epirubicin and cyclophosphamide; and cyclophosphamide, methotrexate, and 5-fluorouracil), there were no deaths or congenital malformations when chemotherapy was given after the first trimester [10].

Although the spontaneous onset of labor and preeclampsia have been reported in patients who received chemotherapy, it is not known whether the incidence of these complications is higher than that in the healthy population, or to what extent chemotherapy affects the underlying disease. Myelosuppression occurring around the time of delivery may put the baby and mother at risk of developing sepsis and hemorrhage; hence, it is recommended that chemotherapy should be avoided at least three weeks before delivery so that maternal blood counts are optimal. Transient leukopenia without infectious sequelae has been reported in neonates born to mothers receiving chemotherapy. Children exposed to chemotherapy in utero are expected to have a higher risk of intrauterine growth retardation; however, the exact incidence is unknown [31-37].

\section{Breastfeeding}

Some studies have suggested that breastfeeding may slightly lower breast cancer risk, especially if breastfeeding is continued for one and a half to two years or if several children are breastfed [38]. A possible reason for this may be that both pregnancy and breastfeeding reduce a woman's total number of lifetime menstrual cycles. However, as not all studies support this notion, more research is needed in this area. 


\section{Chemotherapeutic agents and toxicities}

Pregnancy is a state of increased plasma volume, decreased albumin concentration, decreased gastric motility, and the possible existence of the amniotic sac as a third space. Almost all chemotherapy agents cross the placenta. Therefore, it is reasonable to avoid or reduce chemotherapy drugs for one month before delivery to prevent undue pressure on the newborn's liver and kidneys, which are not able to metabolize or excrete those drugs quickly. Nevertheless, after delivery, cyclophosphamide, methotrexate, and doxorubicin should be avoided as they can enter breast milk.

A French survey found that among women receiving breast cancer chemotherapy 25.2 days before delivery, the incidence of leukopenia was relatively high in their newborns [40]. In a prospective clinical trial, 24 patients were administered fluorouracil, cyclophosphamide, and doxorubicin in the second and third trimesters similar to non-pregnant patients, except in the first trimester [9].

\section{Anthracyclines}

Anthracycline-based chemotherapy seems to be safe during the second and third trimesters. Based on a review of 160 patients who took anthracyclines during pregnancy, Germann et al. suggested that a $<70 \mathrm{mg} / \mathrm{m}^{2}$ dose of doxorubicin per cycle should be given as a short infusion or bolus. The risk of fetal toxicity was increased 30-fold when the dose of doxorubicin per cycle exceeded $70 \mathrm{mg} / \mathrm{m}^{2}(\mathrm{P}=0.037)$ [29]. Maternal exposure to anthracyclines is another long-term risk factor for fetal cardiotoxicity. However, in a comprehensive published review, no case of fetal cardiotoxicity was seen [40].

\section{Taxanes}

Paclitaxel has been used in combination with cisplatin from 28 weeks of pregnancy and in combination with epirubicin from 14 weeks with no fetal or maternal complications [41]. Similarly, docetaxel has also been used both as a single agent and in combination with doxorubicin after 14 weeks of pregnancy in patients with breast cancer with no specific complications [33]. Concerning other antineoplastic agents, vinorelbine in combination with 5fluorouracil has been given to three patients in the second and third trimesters with no adverse effects [34].

\section{Cyclophosphamide}

Cyclophosphamide has been known to cause frequent congenital malformations. The relative risk of congenital disabilities with alkylating agents has been found to be ranging from $4 \%$ to $13 \%$ in late pregnancy [42]. However, it is difficult to distinguish the effects of any one drug, as the patient is exposed to multiple modalities of treatment.

\section{Methotrexate}

Methotrexate is strongly contraindicated because it is a known abortifacient and the leading cause of chemotherapy-related birth defects.

\section{Fluorouracil}

The teratogenicity of fluorouracil remains largely unclear. It has been associated with bony aplasia and hypoplasia, although concurrent exposure to at least 5 rads (0.05 Gy) during 
diagnostic testing cannot be discounted.

\section{Antiemetics}

There is a significant association between corticosteroid use in the first trimester and cleft palate. However, corticosteroids as part of the antiemetic regimen seem to be safe during and after the second trimester. The use of ondansetron during the first trimester does not seem to cause malformations [35-37]. Hence, antiemetic regimens with corticosteroids and ondansetron may be safe during the second and third trimesters of pregnancy.

Granulocyte Colony-stimulating Factor (G-CSF) and Erythropoietin

G-CSF and erythropoietin have been used safely in pregnant patients.

\section{Trastuzumab}

Human epidermal growth factor receptor-2 (HER2) expression is high in embryonic tissues, suggesting that it has a role in embryonic development, and placental transfer of trastuzumab has been observed in animal studies. In a previous study, three patients received trastuzumab: one patient had trastuzumab with weekly vinorelbine, $60 \mathrm{mg} / \mathrm{m}^{2}$, with no adverse effect, whereas the treatment of the other two patients was associated with the development of anhydramnios, which subsided after treatment withdrawal [42].

\section{Hormonal Agents}

Tamoxifen has been shown to be potentially teratogenic in animal studies. Although tamoxifen has safely been given in pregnant patients with metastatic disease without damage to the child, there are reports of birth defects, such as ambiguous genitalia and craniofacial defects. Therefore, the use of tamoxifen is usually delayed until the end of pregnancy. The long-term effects of tamoxifen use and whether it may increase gynecological cancers in daughters (as diethylstilbestrol does) are unknown; in pregnant rats, tamoxifen has been associated with breast cancer in the female offspring. Per updated guidelines, tamoxifen and trastuzumab are contraindicated owing to their reported fatal fetal effects.

\section{Bisphosphonates}

The use of pamidronate was reported in three patients with malignancy-associated hypercalcemia. Two neonates developed transient hypocalcemia but without any developmental problem [40]. It is possible that this hypocalcemia was a result of parathyroid suppression in the neonates due to maternal hypercalcemia, rather than a direct effect of pamidronate on the fetus. Hence, it is recommended that when bisphosphonates are used in pregnant women, the neonatal calcium levels should be carefully monitored.

\section{Termination of pregnancy}

Consideration of pregnancy termination depends on the prognosis of cancer and the treatment options. Termination of pregnancy may allow earlier adjuvant radiotherapy but does not seem to improve prognosis or survival [43]. Even in the advanced stages, elective abortion in combination with castration (bilateral oophorectomy) to decrease the potential hormonal stimulation of breast cancers has not been shown to improve survival.

\section{Prognosis}


Some studies have found that the prognosis in women who were diagnosed as having breast cancer within two years of giving birth was poorer than that in other women with breast cancer; however, other studies did not observe this difference [44-45]. More research is needed in this area.

Pregnancy During Breast Cancer

Despite the responsiveness of breast tissue to hormonal stimulation in the pregnant and lactation state (when controlled for patient age and disease stage), the prognosis does not seem to differ from that in non-pregnant patients of the same age and stage of disease [46]. There is a school of thought among doctors that terminating the pregnancy may help slow the course of more advanced breast cancers; however, conducting research in this area is difficult and very few quality studies have been performed. The studies conducted to date have not shown that terminating the pregnancy improves a woman's prognosis. Moreover, studies have not demonstrated that the types of treatment delays that are sometimes needed during pregnancy have an effect on breast cancer outcome; however, again, this has proven to be a difficult area to study.

\section{Pregnancy After Breast Cancer Treatment}

Certain chemotherapeutic drugs may affect a woman's fertility, but many women will still be able to become pregnant after treatment. The percentage of patients who have full-term pregnancies after a breast cancer diagnosis is minuscule. Among women younger than 45 years at diagnosis, only $3 \%$ subsequently give birth to a live infant, and among women younger than 35 years at diagnosis, $8 \%$ have full-term pregnancies [47]. Various factors may contribute to the low pregnancy rate, and among them is the increased chance of spontaneous abortions, occurring in nearly $25 \%$ of pregnancies [47]. It is uncertain whether women with a history of breast cancer have an increased risk of cancer recurrence after becoming pregnant. Most of the previous studies found that pregnancy does not increase the risk of recurrence after successful treatment of breast cancer. The link between estrogen levels and growth of breast cancer cells is well established, and because of this link, the general advice for breast cancer survivors is to wait at least two years before attempting to become pregnant. This would allow any early relapse to be diagnosed, which could affect a woman's decision to become pregnant in the future. Moreover, this advice is not based on evidence from clinical trials, and the decision is influenced by many factors, including the woman's desire for further pregnancies, age, statistical risk of an early relapse from cancer, and potential impact of estrogens on the risk of breast cancer recurrence.

Hormonal therapeutic drugs, such as tamoxifen, could affect a developing fetus and should be avoided. There is no evidence that a previous history of breast cancer in the mother has any effect on the baby. The survival of pregnant and non-pregnant patients with breast cancer has been reported to be equal. The prognosis does not improve for pregnant patients who aborted and received standard treatment for the disease.

\section{Multidisciplinary approach}

Genetic counseling is recommended for all pregnant patients with breast cancer, as one casecontrol study showed that a family history of breast cancer was three times more common in pregnant and lactating women than in non-pregnant women with breast cancer [48]. Further studies are needed to explore the potential relationship between mutations of individual breast cancer susceptibility genes and the subsequent development of breast cancer during 
pregnancy. In carriers of breast cancer susceptibility genes 1 and 2 (BRCA1/BRCA2) mutations, pregnancy also increases the risk of breast cancer. Cullinane et al. showed that in BRCA1 carriers, the risk of breast cancer decreases significantly after four or more births, whereas in BRCA2 carriers, parity after two or more births increases the risk of breast cancer (by 1.5 -fold compared to nulliparous women) [49]. In BRCA2 mutation carriers, the breast cancer risk within two years after delivery is $70 \%$ higher than that in nulliparous women. Cancer during pregnancy is a stressful situation; therefore, measures should be taken to provide psychological support during treatment and delivery to the patient and her family.

\section{Monitoring of pregnancy}

Before starting chemotherapy, fetal ultrasonography should be performed to ensure that the fetus appears normal, and the gestational age and expected date of delivery should be estimated. Ultrasonography should be performed before every cycle of chemotherapy to assess fetal growth. Any fetal abnormalities and pregnancy-related complications should be treated based on the standard recommendations.

\section{Delivery and postpartum}

Studies have reported that women with hematological and non-hematological malignancies who received chemotherapy are more likely to deliver prematurely. When fetal maturation is sufficient, labor can be induced or a cesarean section can be performed in a hospital with adequate neonatal support. The timing of the delivery can be optimized with the treatment of the mother's breast cancer. It is recommended that the timing of delivery be approximately three weeks after the last dose of chemotherapy [39]. The placenta should be sent for histopathological examination to rule out the rare possibility of placental metastases.

\section{Breastfeeding}

Breastfeeding during chemotherapy and hormonal therapy is contraindicated, as most drugs can be excreted in breast milk. Nevertheless, there is no evidence to support the belief that women who have completed treatment for breast cancer cannot breastfeed safely from the unaffected breast. One study showed that bilateral lactation could be possible after conservative surgery and radiation therapy in approximately $25 \%$ of women [50].

\section{Future pregnancy}

Women should be advised that premature menopause might result from breast cancer treatments, especially if chemotherapy is given to patients older than 30 years. It is recommended that pregnancy should be delayed for at least two years after treatment completion. The recommendation for future conception may be based on the prognosis of individual women. Women with Stage IV disease should not consider pregnancy, and women with Stage III disease should avoid becoming pregnant for at least five years after treatment. Women with recurrent Stage I or II tumors should not consider conception because of the intensity of the required treatment and the poor prognosis.

\section{Conclusions}

As more women postpone childbearing until middle age, the incidence of breast cancer in pregnancy is increasing and delays in diagnosis are more common. It is emphasized that women should undergo periodic breast examinations during prenatal visits. Among the investigations, mammography is of limited value because of pregnancy-induced changes in the breast and some amount of associated risk to the developing fetus in the later part of pregnancy. Fine-needle aspiration or core needle biopsy has emerged as the diagnostic tool of choice in pregnant women with breast cancer. Furthermore, ultrasonography is also a useful 
diagnostic tool, along with a clinical examination.

Regarding the treatment options, depending on the time of diagnosis, mastectomy and axillary dissection are the traditional treatments of choice. Lumpectomy followed by irradiation can be done after delivery. Radiation during pregnancy is best avoided to prevent the risk to the fetus. In addition to their long-term effects, chemotherapy and hormonal therapy have largely been proven to be safe after the second trimester and in the third trimester.

We recommend providing genetic counseling to all patients with pregnancy-associated breast cancer to serve as a guide for future pregnancies.

\section{Additional Information}

\section{Disclosures}

Conflicts of interest: In compliance with the ICMJE uniform disclosure form, all authors declare the following: Payment/services info: All authors have declared that no financial support was received from any organization for the submitted work. Financial relationships: All authors have declared that they have no financial relationships at present or within the previous three years with any organizations that might have an interest in the submitted work. Other relationships: All authors have declared that there are no other relationships or activities that could appear to have influenced the submitted work.

\section{References}

1. Middleton LP, Amin M, Gwyn K, et al.: Breast carcinoma in pregnant women. Assessment of clinicopathologic and immunohistochemical features. Cancer. 2003, 98:1055-60. 10.1002/cncr.11614

2. Woo JC, Yu T, Hurd TC: Breast cancer in pregnancy: a literature review . Arch Surg. 2003, 138:91-98. 10.1001/archsurg.138.1.91

3. Zemlickis D, Lishner M, Degendorfer P, et al.: Maternal and fetal outcome after breast cancer in pregnancy. Am J Obstet Gynecol. 1992, 166:781-87. 10.1016/0002-9378(92)91334-7

4. Bonnier P, Romain S, Giacalone PL, et al.: Clinical and biologic prognostic factors in breast cancer diagnosed during postmenopausal hormone replacement therapy. Obstet Gynecol. 1995, 85:11-17. 10.1016/0029-7844(94)00324-7

5. Costa SD, Lange S, Klinga K, et al.: Factors influencing the prognostic role of oestrogen and progesterone receptor levels in breast cancer-results of the analysis of 670 patients with 11 years of follow-up. Eur J Cancer. 2002, 38:1329-34. 10.1016/S0959-8049(02)00067-9

6. Opdahl S, Alsaker K, Janszky I, et al.: Joint effects of nulliparity and other breast cancer risk factors. Br J Cancer. 2011, 105:731-36. 10.1038/bjc.2011.286

7. Travis RC, Key TJ: Oestrogen exposure and breast cancer risk. Breast Cancer Res. 2003, 5:23947. 10.1186\%2Fbcr628

8. Scott-Connor CE, Schorr S: The diagnosis and management of breast problems during pregnancy and lactation. Am J Surg. 1995, 170:401-405. 10.1016/S0002-9610(99)80313-4

9. Berry DL, Theriault RL, Holmes FA, et al.: Management of breast cancer during pregnancy using a standardized protocol. J Clin Oncol. 1999, 17:855-61. 10.1200/JCO.1999.17.3.855

10. Patel SJ, Reede DL, Katz DS, et al.: Imaging the pregnant patient for nonobstetric conditions: algorithms and radiation dose considerations. Radiographics. 2007, 27:1705-22.

10.1148/rg.276075002

11. Nicklas AH, Baker ME: Imaging strategies in the pregnant cancer patient. Semin Oncol. 2000 Dec, 27:623-32.

12. Hogge JP, De Paredes ES, Magnant CM, Lage J: Imaging and management of breast masses during pregnancy and lactation. Breast J. 1999, 5:272-83. 10.1046/j.1524-4741.1999.98077.x

13. Lyman GH, Giuliano AE, Somerfield MR, et al.: American Society of Clinical Oncology guideline recommendations for sentinel lymph node biopsy in early-stage breast cancer. J Clin Oncol. 2005, 23:7703-20. 10.1200/JCO.2005.08.001 
14. De Santis M, Straface G, Cavaliere AF, et al.: Gadolinium periconceptional exposure: pregnancy and neonatal outcome. Acta Obstet Gynecol Scand. 2007, 86:99-101. 10.1080/00016340600804639

15. Yang WT, Dryden MJ, Gwyn K, et al.: Imaging of breast cancer diagnosed and treated with chemotherapy during pregnancy. Radiology. 2006, 239:52-60. 10.1148/radiol.2391050083

16. Kal HB, Struikmans H: Radiotherapy during pregnancy: fact and fiction . Lancet Oncol. 2005, 6:328-33. 10.1016/S1470-2045(05)70169-8

17. Liberman L, Giess CS, Dershaw DD, et al.: Imaging of pregnancy-associated breast cancer . Radiology. 1994, 191:245-48. 10.1148/radiology.191.1.8134581

18. Hamaoka T, Madewell JE, Podoloff DA, et al.: Bone imaging in metastatic breast cancer . J Clin Oncol. 2004, 22:2942-53. 10.1200/JCO.2004.08.181

19. Ring AE, Smith IE, Ellis PA: Breast cancer and pregnancy. Ann Oncol. 2005, 16:1855-60. 10.1093/annonc/mdi388

20. Peng KW, Lei Z, Xiao TH, et al.: First trimester caesarean scar ectopic pregnancy evaluation using MRI. Clin Radiol. 2014, 69:123-29. 10.1016/j.crad.2013.07.021

21. Mitre BK, Kanbour AI, Mauser N: Fine needle aspiration biopsy of breast carcinoma in pregnancy and lactation. Acta Cytol. 1997, 41:1121-30. 10.1159/000332798

22. Weisz B, Schiff E, Lishner M: Cancer in pregnancy: maternal and fetal implications. Hum Reprod Update. 2001, 7:384-93. 10.1093/humupd/7.4.384

23. Amant F, Deckers S, Van Calsteren K, et al.: Breast cancer in pregnancy: recommendations of an international consensus meeting. Eur J Cancer. 2010, 46:3158-68.

10.1016/j.ejca.2010.09.010

24. Moran BJ, Yano H, Al Zahir N, Farquharson M: Conflicting priorities in surgical intervention for cancer in pregnancy. Lancet Oncol. 2007, 8:536-44. 10.1016/S1470-2045(07)70171-7

25. Mabry H, Giuliano AE: Sentinel node mapping for breast cancer: progress to date and prospects for the future. Surg Oncol Clin N Am. 2007, 16:55-70.

26. Hickey M, Peate M, Saunders CM, Friedlander F: Breast cancer in young women and its impact on reproductive function. Hum Reprod Update. 2009, 15:323-39. 10.1093/humupd/dmn064

27. Morrow M, Strom EA, Bassett LW, et al.: Standard for breast conservation therapy in the management of invasive breast carcinoma. CA Cancer J Clin. 2002, 52:277-300.

10.3322/canjclin.52.5.277

28. Van Calsteren K, Verbesselt R, Ottevanger N, et al.: Pharmacokinetics of chemotherapeutic agents in pregnancy: a preclinical and clinical study. Acta Obstet Gynecol Scand. 2010, 89:1338-45. 10.3109/00016349.2010.512070

29. Germann N, Goffinet F, Goldwasser F: Anthracyclines during pregnancy: embryo-fetal outcome in 160 patients. Ann Oncol. 2004, 15:146-50. 10.1093/annonc/mdh009

30. Nugent P, O'Connell TX: Breast cancer and pregnancy. Arch Surg. 1985, 120:1221-24. 10.1001/archsurg.1985.01390350007001

31. Cardonick E, Bhat A, Gilmandyar D, Somer R: Maternal and fetal outcomes of taxane chemotherapy in breast and ovarian cancer during pregnancy: case series and review of the literature. Ann Oncol. 2012, 23:3016-23. 10.1093/annonc/mds170

32. Potluri V, Lewis D, Burton GV: Chemotherapy with taxanes in breast cancer during pregnancy: case report and review of the literature. Clin Breast Cancer. 2006, 7:167-70. 10.3816/CBC.2006.n.029

33. Mir O, Berveiller P, Goffinet F, et al.: Taxanes for breast cancer during pregnancy: a systematic review. Ann Oncol. 2010, 21:425-26. 10.1093/annonc/mdp517

34. Cuvier C, Espie M, Extra JM, Marty M: Vinorelbine in pregnancy. Eur J Cancer. 1997, 33:16869. 10.1016/S0959-8049(96)00356-5

35. Williams S, Schilsky R: Antineoplastic drugs administered during pregnancy. Semin Oncol. 2000, 27:618-22.

36. Siu SS, Yip SK, Cheung CW, Lau TK: Treatment of intractable hyperemesis gravidarum by ondansetron. Eur J Obstet Gynecol Reprod Biol. 2002, 105:73-74. 10.1016/S0301-

2115(02)00116-1

37. Sullivan CA, Johnson CA, Roach H, et al.: A pilot study of intravenous ondansetron for hyperemesis gravidarum. Am J Obstet Gynecol. 1996, 174:1565-68. 10.1016/S00029378(96)70607-5

38. Cohen JM, Hutcheon JA, Julien SG, et al.: Insufficient milk supply and breast cancer risk: a systematic review. PLoS One. 2009, 4:e8237. 10.1371\%2Fjournal.pone.0008237 
39. Giacalone PL, Laffargue F, Bénos P: Chemotherapy for breast carcinoma during pregnancy: a French national survey. Cancer. 1999, 86:2266-72. 10.1002/(SICI)1097-

0142(19991201)86:11<2266::AID-CNCR14>3.3.CO;2-Z

40. Kelly HL, Collichio FA, Dees EC: Concomitant pregnancy and breast cancer: options for systemic therapy. Breast Dis. 2006, 23:95-101. 10.3233/BD-2006-23113

41. Loibl S: New therapeutic options for breast cancer during pregnancy. Breast Care (Basel). 2008, 3:171-76. 10.1159/000136002

42. Gucalp R, Knight R: Pamidronate for cancer-associated hypercalcemia-Reply . Arch Intern Med. 1995, 155:642-45. 10.1001/archinte.1995.00430060105016

43. Peccatori FA, Azim HA Jr, Orecchia R, et al.: Cancer, fertility and pregnancy: ESMO Clinical Practice Guidelines for diagnosis, treatment and follow-up. Ann Oncol. 2013, 24:vi160-70. 10.1093/annonc/mdt199

44. Rodriguez AO, Chew H, Cress R, et al.: Evidence of poorer survival in pregnancy-associated breast cancer. Obstet Gynecol. 2008, 112:71-78. 10.1097/AOG.0b013e31817c4ebc

45. Beadle BM, Woodward WA, Middleton LP, et al.: The impact of pregnancy on breast cancer outcomes in women $\leqslant 35$ years. Cancer. 2009, 115:1174-84. 10.1002/cncr.24165

46. Hartman EK, Eslick GD: The prognosis of women diagnosed with breast cancer before, during and after pregnancy: a meta-analysis. Breast Cancer Res Treat. 2016, 160:347-60. 10.1007/s10549-016-3989-3

47. Collaborative Group on Hormonal Factors in Breast Cancer: Breast cancer and breastfeeding: collaborative reanalysis of individual data from 47 epidemiological studies in 30 countries, including 50302 women with breast cancer and 96973 women without the disease. Lancet. 2002, 360:187-95. 10.1016/S0140-6736(02)09454-0

48. Amant F, Loibl S, Neven P, Van Calsteren K: Breast cancer in pregnancy. Lancet. 2012, 379:570-79. 10.1016/S0140-6736(11)61092-1

49. Cullinane CA, Lubinski J, Neuhausen SL, et al.: Effect of pregnancy as a risk factor for breast cancer in BRCA1/BRCA2 mutation carriers. Int J Cancer. 2005, 117:988-91. 10.1002/ijc.21273

50. Higgins S, Haffty BG: Pregnancy and lactation after breast-conserving therapy for early stage breast cancer. Cancer. 1994, 73:2175-80. 